\title{
Uma experiência de inserção de Linguagem de Programação no Ensino Médio Integrado à Educação Profissional Técnica a partir da flexibilização curricular
}

\section{An experience of insertion of Programming Language in High School Integrated with Technical Professional Education based on curricular flexibility}

\author{
Una experiencia de inserción de Lenguaje de Programación en Bachillerato \\ Integrado con Educación Técnica Profesional basada en la flexibilidad \\ curricular
}

Fria, Bruno Lopes de ${ }^{1}$ (Diamantina, MG, Brasil) ORCID ID: https://orcid.org/0000-0002-8560-0034 Alves, Janainne Nunes ${ }^{2}$ (Diamantina, MG, Brasil) ORCID ID: https://orcid.org/0000-0002-3388-7053

Costa, Claudiane Moreira ${ }^{3}$ (Diamantina, MG, Brasil) ORCID ID: http://orcid.org/0000-0001-5473-5246

\begin{abstract}
Resumo
O princípio de flexibilização curricular pressupõe inovações nos projetos pedagógicos dos cursos para atender às novas realidades sociais, entre as quais destacamos a imersão tecnológica contemporânea. Nesse sentido, apresentamos uma ementa curricular como aporte teórico-metodológico para o desenvolvimento de competências relacionadas ao aprendizado de uma Linguagem de Programação (i.e pensamento algorítmico e resolução de problemas do mundo real em linguagem computacional) enquanto unidade curricular aplicada a diferentes perfis profissionais do nível médio. A ementa foi consolidada por meio de uma abordagem holística fundamentada em critérios como coerência, exequibilidade e curva de aprendizagem que proporcionem um caráter globalizante em função da heterogeneidade das formações. A viabilidade da proposta foi sugerida por formulários de aceitação em escala e observações realizadas no contexto da sala de aula nos cursos integrados em Informática, Meio Ambiente e Teatro do IFNMG/Diamantina. O nível de aprendizagem dos estudantes foi classificado em uma escala de 1 = muito fácil a 5 = muito difícil, onde obtivemos média de 2,57 . Os resultados indicam que na linguagem adotada, Python, nenhum quesito foi considerado particularmente difícil, o que pode ser relacionado à sua sintaxe compacta e limpa, se comparada a outras linguagens de programação. A aplicação preliminar da proposta no IFNMG demonstrou boa interação dos estudantes com a Python, assim como assimilação e capacidade de ressignificar o conteúdo adquirido através da construção de propostas de trabalho conjunto e aplicação profissional.
\end{abstract}

Palavras-chave: Perfil profissional. Competências. Flexibilidade curricular. Tecnologia.

\begin{abstract}
The principle of curricular flexibility presupposes innovations in the pedagogical projects of the courses to meet the new social realities, among which we highlight the contemporary technological immersion. In this sense, we present a curricular menu as a theoretical-methodological contribution to the development of skills related to the learning of a Programming Language (ie algorithmic thinking and problem solving in the real world in computational language) as a curricular unit applied to different professionais profiles at the level medium. The menu was consolidated through a holistic approach based on criteria such as coherence, feasibility and a learning curve that provide a global character due to the heterogeneity of training. The feasibility of the proposal was suggested by scale acceptance forms and observations made in the classroom context in the IFNMG/Diamantina integrated courses in Informatics, Environment and Theater. The students' level of learning was rated on a scale of $1=$ very easy to 5 = very difficult, where we obtained an average of 2.57. The results indicate that in the adopted language, Python, no item was considered particularly difficult, which can be related to its compact and

\footnotetext{
1 Professor de Informática no Instituto Federal do Norte de Minas Gerais (IFNMG). bruno.lopes@ifnmg.edu.br

2 Professora do Instituto Federal de Educação, Ciência e Tecnologia do Norte de Minas Gerais (IFNMG). janainne.alves@ifnmg.edu.br.

3 Técnica em assuntos educacionais do Instituto Federal de Educação, Ciência e Tecnologia do Norte de Minas Gerais (IFNMG). claudiane.costa@ifnmg.edu.br.
} 
clean syntax, when compared to other programming languages. The preliminary application of the proposal at the IFNMG demonstrated good interaction between students and Python, as well as assimilation and the ability to reframe the acquired content through the construction of proposals for joint work and professional application.

Keywords: Professional Profile. Skills. Curricular flexibility. Technology.

\section{Resumen}

El principio de flexibilidad curricular presupone innovaciones en los proyectos pedagógicos de los cursos para afrontar las nuevas realidades sociales, entre las que destacamos la inmersión tecnológica contemporánea. En este sentido, presentamos un menú curricular como un aporte teórico-metodológico al desarrollo de habilidades relacionadas con el aprendizaje de un Lenguaje de Programación (es decir, pensamiento algorítmico y resolución de problemas en el mundo real en lenguaje computacional) como unidad curricular aplicada a diferentes perfiles profesionales a nivel medio. El menú se consolidó a través de un enfoque holístico basado en criterios como la coherencia, la viabilidad y una curva de aprendizaje que otorgan un carácter global debido a la heterogeneidad de la formación. La viabilidad de la propuesta fue sugerida por formularios de aceptación de escala y observaciones realizadas en el contexto del aula en los cursos integrados IFNMG/Diamantina en Informática, Medio Ambiente y Teatro. El nivel de aprendizaje de los estudiantes se calificó en una escala de 1 = muy fácil a 5 = muy difícil, donde obtuvimos una media de 2,57. Los resultados indican que en el lenguaje adoptado, Python, ningún ítem fue considerado particularmente difícil, lo cual puede estar relacionado con su sintaxis compacta y limpia, en comparación con otros lenguajes de programación. La aplicación preliminar de la propuesta en el IFNMG demostró una buena interacción entre los estudiantes y Python, así como la asimilación y la capacidad de replantear los contenidos adquiridos a través de la construcción de propuestas de trabajo conjunto y aplicación profesional.

Palavras-Clave: Perfil profesional. Habilidades. Flexibilidad curricular. Tecnología.

\section{Introdução}

A aprendizagem é compreendida como um processo experimental construído por intermédio da relação do indivíduo com a cultura, o qual se manifesta através de modificações comportamentais. Trata-se de um fenômeno complexo que envolve aspectos cognitivos, emocionais, orgânicos, psicossociais e culturais (TABILE e JACOMETO, 2017). E engloba um conjunto de saberes e experiências que a cultura proporcionou aos sujeitos individuais e coletivos que constroem a sociedade ao longo de sua existência (FERRETI e SILVA, 2017). Ademais, sob o ponto de vista freiriano, a educação é pautada nas ações do homem como ser aberto ao mundo, de forma a captá-lo e compreendê-lo, atuando de acordo com suas finalidades, a fim de transformá-lo (FREIRE, 1969).

Partindo dessa perspectiva educacional, materializada como um processo social de transformação do meio, e tendo como preceito a lei de diretrizes e bases (LDB) é imprescindível considerar a influência tecnológica na sociedade contemporânea, bem como suas relações com as comunicações, o trabalho e o desenvolvimento de práticas pedagógicas que assegurem aos estudantes instrumentos para progredir no cenário emergente (BRASIL,1996; FEIJÓ, XAVIER \& ARRAIS NETO, 2019). 
Nos últimos anos, o uso das tecnologias tem se apresentado cada vez mais presente no cotidiano dos estudantes do ensino médio. Jovens, que integram os chamados "nativos digitais", uma geração nascida na era da internet, que teve seus modos de interação social, experiências e compreensão da informação completamente modificados pelo meio digital (PALFREY e GASSER, 2011).

Contudo, é de nosso conhecimento que a grande maioria dos jovens, que compõem essa geração, dissipa boa parte do tempo consumindo passivamente essas tecnologias (seja através das redes sociais ou dos jogos), o que não os torna fluentes no universo tecnológico. Restringindo-se a bons consumidores de informações produzidas e filtradas através da tecnologia e, portanto, inaptos a compreender 0 pensamento computacional dos recursos tecnológicos consumidos (i.e reconhecimento de padrões, decomposição de problemas, abstração de detalhes, identificação e organização passo a passo necessária para resolver um problema através de um algoritmo) e aplicá-los no cotidiano.

Essa realidade tem levado educadores a ponderarem sobre a influência das constantes mudanças da sociedade, onde destacamos a inovação tecnológica, sobre o processo educacional e motivado a busca por estratégias de ensino emancipatórias, capazes de intensificar o desempenho dos estudantes (DA SILVA e PORTILHO, 2018). Discurso que tem como pauta a (re) construção curricular, visto seu potencial em empreender no âmbito escolar.

Ainda sob essa concepção, é importante inferir que um processo de ensino e aprendizagem emancipatório, conceitualmente tido como integral, possibilita que os sujeitos desenvolvam suas potencialidades e para tal, apresenta uma relação íntima com as pretensões e necessidades sociais. Consequentemente, isso requer currículos escolares estruturados de modo a permitir que o indivíduo transcenda o conhecimento elementar e explore sua capacidade em produzir, acessar e operar a informação (ANTUNES E PADILHA, 2000; DA SILVA E FLACH; 2017; FLACH e SCHLESENER, 2017).

Logo, compreende-se que a referência curricular às unidades promotoras do raciocínio lógico, do pensamento crítico e reflexivo, se apresenta como fundamental ao preparo dos jovens em todas as esferas profissionais vislumbradas pelo mundo do trabalho, bem como vivência e convivência em sociedade, com consequente intervenção na realidade (FRIGOTTO, CIAVATTA E RAMOS, 2005; 
PINTO et al., 2019).

Sob o pressuposto da reestruturação dos currículos aliado à crescente implementação das tecnologias da informação e comunicação em todos os segmentos profissionais e sociais, este estudo traz uma proposta de ementa curricular estruturada para a oferta de Linguagem de Programação (LP) a eixos tecnológicos diversificados ${ }^{4}$. Ou seja, direcionada ao atendimento dos múltiplos perfis profissionais dos cursos do Ensino Médio Integrado à Educação Profissional e Tecnológica (EMIEPT). Complementarmente, com o propósito de delinear uma melhor compreensão sobre a viabilidade dessa proposta (ementa curricular), apresentamos uma análise preliminar obtida a partir de investigações sobre a perspectiva e aceitação dos estudantes num primeiro contato com a LP, posto que esta foi ofertada como unidade curricular aos cursos EMIEPT em Informática, Meio Ambiente e Teatro do IFNMG/Diamantina-MG.

\section{Fundamentação teórica}

Os progressivos avanços das tecnologias digitais de informação e comunicação têm impactado na organização econômica, social e cultural, exercendo uma influência direta sobre as interações sociais, as transações comerciais, bem como a maneira de acessar informação (VALENTE, 2016). A cultura digital se insere celeremente em todos as seções sociais, o que torna o desenvolvimento de aptidões nessa área essenciais ao bom desempenho profissional e atuação social. Percepção, que nos coloca diante de inevitáveis reflexões acerca da estrutura curricular e práticas educacionais.

Complementarmente, observa-se que a utilização das tecnologias digitais pelos estudantes do ensino médio tem se limitado à compreensão e exploração das ferramentas de escritório e ao acesso à informação e comunicação, em particular de redes sociais e jogos. O que ratifica a necessidade de ponderações sobre a abordagem tecnológica no contexto escolar, de maneira que se permita aos estudantes realmente usufruir e explorar as tecnologias da informação e comunicação (BEHRENS e JOSÉ, 2001; VALENTE, 2016).

A partir dessa perspectiva, depreende-se que um processo de ensino e

4 Os cursos ofertados de EMIEPT do IFNMG campus Diamantina estão inseridos nos Eixos Tecnológicos de Ambiente e saúde, Informática e comunicação, Produção Cultural e Design com as seguintes habilitações respectivamente: Técnico em Meio Ambiente, Técnico em Informática e Técnico em Teatro. 
aprendizagem atento às mudanças e necessidades sociais, incita a alusão aos recursos tecnológicos e abarca estímulos e capacidades cognitivas capazes de ampliar o pensamento lógico e a visão de mundo dos estudantes. Programar, por exemplo, tem como preceitos o processo de desenvolver um raciocínio, analisar e criar uma solução em meio computacional.

Ademais, observa-se uma propensão significativa de muitos países a aderirem à incorporação curricular de disciplinas associadas ao letramento digital (habilidade de usar computadores e tecnologias de maneira eficientemente, abrange desde funcionalidades elementares até o uso de uma linguagem de programação necessária para resolver um problema por meio de um processo algorítmico). Destaca-se que o ensino de programação integra o currículo da educação básica (EB) em praticamente todos os países da Comunidade Europeia e dos EUA. Por sua vez, a Comissão Europeia publicou o relatório European Schoolnet (2014), documento que teve como referência a situação de vinte países europeus, entre os quais treze países já incluíram o ensino de programação nas disciplinas obrigatórias do ensino infantil ao nono ano, o equivalente ao nosso Ensino Fundamental (SCAICO, 2013; GARDNER, CHEN \& MORAN, 2009). Países como Lituânia, Portugal, Grécia, Irlanda e Bulgária adotam metodologias semelhantes e o ensino de programação também é abordado ao longo do ensino fundamental (SCHOOLNET, 2014).

No Brasil, as disciplinas relacionadas ao ensino de programação na EB se restringem, em sua grande maioria, aos cursos profissionalizantes com formação específica em informática e áreas afins. Todavia, as Diretrizes Curriculares Nacionais (DCN's) para a educação profissional tecnológica (EPT) já sinalizam para o desenvolvimento de abordagens que integrem o conhecimento escolar às dimensões sociais, processo que abrange fundamentos científico-tecnológicos:

Os cursos de Educação Profissional Técnica de Nível Médio têm por finalidade proporcionar ao estudante conhecimento, saberes e competências profissionais necessários ao exercício profissional e da cidadania, com base nos fundamentos científico-tecnológicos, sócio-históricos e culturais (BRASIL, 2012, p. 12).

Ainda refletindo sobre esses princípios, o artigo 14 das DCN's para EPT de nível médio, regulamenta que o currículo deve proporcionar aos estudantes um diálogo com os diversos campos do trabalho, da ciência, da tecnologia e da cultura, designados como essenciais na formação. Além de fornecer elementos para 


\section{Revista Labor}

Programa de Pós-graduação em Educação, Universidade Federal do Ceará

Fortaleza-CE-Brasil

compreensão e discussão das relações sociais de produção e trabalho, assim como as especificidades históricas na sociedade contemporânea. De forma a auxiliar o exercício profissional com competência, idoneidade intelectual e tecnológica, autonomia e responsabilidade (BRASIL, 1996; BRASIL, 2012; BRASIL, 2017).

Enquanto promotores da EPT, os Institutos Federais de Educação, criados por meio da Lei o 11.892 de 29 de dezembro de 2008, possuem uma proposta formativa edificada a partir dos diferentes fundamentos da vida que estruturam a prática social. Proposição que se mostra afim ao processo educacional emancipatório e que em conjunto com a autonomia dessas instituições permite a implementação de uma organização curricular flexível (BRASIL, 2012; RAMOS, 2008; COSTA JÚNIOR et al., 2017, CONIF/FDE, 2018).

Tendo essa concepção formativa como alicerce, o IFNMG/Diamantina instituiu uma relação particular entre a oferta disciplinar e as interfaces sociais que compreendem a formação dos estudantes nos cursos EMIEPT por meio de três núcleos (figura 1): o Núcleo Básico (aborda os conteúdos da Base Nacional Comum Curricular/Nível Médio), o Núcleo Tecnológico (aborda os conteúdos diretamente relacionados ao perfil profissional do egresso) e o Núcleo Integrador (conteúdo diversificado, flexível).

Figura 1 - Representação esquemática da organização curricular no IFNMG/Diamantina

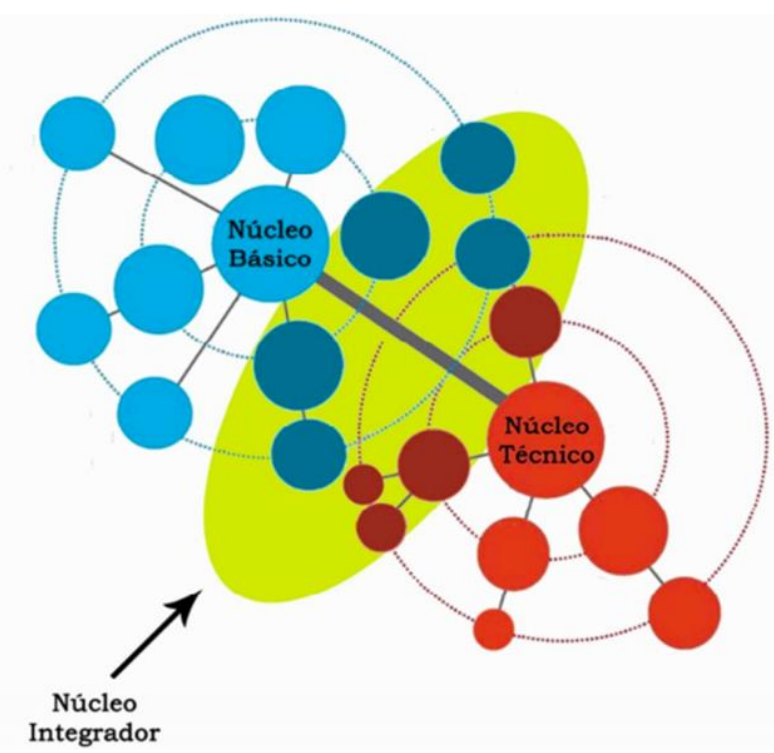

Fonte: IFNMG (2017)

No sentido de ampliar as percepções que abarcam os aspectos 
Programa de Pós-graduação em Educação, Universidade Federal do Ceará

Fortaleza-CE-Brasil

organizacionais e conceitos relacionados aos currículos EMIEPT do

IFNMG/Diamantina, os próximos parágrafos detalham os núcleos estruturantes (figura

1) que os compõem.

Núcleo Técnico ou Tecnológico:

[...] um espaço da organização curricular ao qual se destinam os componentes curriculares que tratam dos conhecimentos e habilidades inerentes à educação técnica e que possuem maior ênfase tecnológica e maior área de integração com os demais componentes curriculares do curso

Núcleo Básico: em relação ao perfil profissional do egresso (IFNMG, 2017, p.33).

Núcleo Integrador:

[...] um espaço da organização curricular ao qual se destinam os componentes curriculares que tratam dos conhecimentos e habilidades inerentes à educação básica e que possuem menor ênfase tecnológica. [...] constituído essencialmente a partir dos conhecimentos e habilidades nas áreas de linguagens e seus códigos, ciências humanas, matemática e ciências da natureza, que têm por objetivo desenvolver o raciocínio lógico, a argumentação, a capacidade reflexiva, a autonomia intelectual (IFNMG, 2017, p.34).

[...] um espaço da organização curricular ao qual se destinam as disciplinas que tratam dos conhecimentos e habilidades inerentes à educação básica e técnica, que possuem maior área de integração com as demais disciplinas do curso em relação ao perfil do egresso bem como as formas de integração [...]. O núcleo integrador compreende fundamentos científicos, sociais, organizacionais, econômicos, políticos, culturais, ambientais, estéticos e éticos que alicerçam as tecnologias e a contextualização do eixo tecnológico no sistema de produção social (IFNMG, 2017, p. 34).

\section{O Núcleo Integrador traz consigo especificidades que permitem}

construções educacionais singulares, visto que as unidades ofertadas neste núcleo podem ser construídas e/ou reformuladas semestralmente por estudantes e professores, e, portanto, constantemente articuladas a novos estímulos e aprendizagens, como a LP (IFNMG, 2017).

Um diferencial importante na sistematização curricular do IFNMG/Diamantina refere-se ao processo de matrícula nas unidades curriculares ofertadas no Núcleo Integrador. Sobre esse aspecto é imprescindível destacar que as unidades podem ser cursadas por estudantes pertencentes a eixos tecnológicos e séries distintos.

Condição que cria um ambiente propício à convivência de estudantes matriculados no primeiro e/ou segundo ano dos cursos técnicos em Informática e estudantes matriculados no primeiro ano do curso técnico em Meio ambiente, por exemplo. Fomentando assim, uma troca de experiências abundante, em virtude da 
heterogeneidade dos perfis.

Denota-se que a perspectiva educacional do IFNMG/Diamantina contempla um processo que conduz o estudante a melhores entendimentos e enfrentamento dos desafios inerentes às diferentes realidades. Devido ao estímulo à formação de profissionais flexíveis "no sentido de terem a base que possibilita uma rápida compressão e domínio das transformações e inovações produtivas" (CIAVATTA e RAMOS, 2012). Aspectos que permitiram a abordagem de LP como uma proposta de diálogo entre a tecnologia e as diferentes formações técnico-profissionais dos cursos EMIEPT por meio da ementa aqui apresentada.

\section{Metodologia}

A natureza da investigação adotada aborda aspectos quali-quantitativos e foi realizada sob as perspectivas da matriz curricular do EMIEPT do IFNMG/Diamantina.

O estudo teve início com o desenvolvimento de uma ementa propícia para a abordagem de LP em turmas heterogêneas. Nesse seguimento, a escolha da LP ideal para aplicação no EMIEPT, assim como o processo de construção da ementa foram consolidados de acordo com as convicções de Farooq et al. (2012), que têm como pressupostos os seguintes itens:

1) Sintaxe simples. Um dos grandes desafios para um novo programador é familiarizar-se com a sintaxe da linguagem em que ele está programando. Uma LP simples e clara, ajudará um programador iniciante na compreensão rápida da linguagem. Quesito de extrema relevância no caso apresentado, onde o ensino é destinado a uma extensão de perfis técnico-profissionalizantes.

2) Ausência de pré-requisitos. Uma consideração relevante na seleção da LP para estudantes da educação básica, é a ausência de conhecimentos prévios em programação.

3) Fácil implementação. Um dos principais desafios em termos de introduzir uma LP na educação básica é a dificuldade inerente ao aprender a programar. Preocupação anteriormente observada a nível universitário, onde o ensino de LP já se encontra implementado (MITCHEL RESNICK, 2009; DA SILVA et al., 2015). Por isso, a LP para este primeiro contato deve ser simples, com forma recortada (indentação), estruturada, descomplicada, com boa implementação e legibilidade do código. 
4) Linguagem de programação de scripts. São desejáveis linguagens de script projetadas para feedback rápido para o usuário, melhorando a identificação e correção dos erros.

5) Fácil transição para outras LP. Existe um consenso entre os especialistas, de que a primeira linguagem de programação deve ser de alto nível (próximo à linguagem humana), e os conhecimentos adquiridos devem ser úteis ao aprendizado de novas linguagens de programação populares na academia e no mercado de trabalho, como Java e $\mathrm{C}++$.

6) Alinhamento com o currículo escolar. Introdução de conceitos de forma gradual e vinculada a outras disciplinas do currículo escolar.

No processo de escolha de conteúdos para elaboração da ementa, foram considerados aspectos como coerência e exequibilidade, de maneira a proporcionar um caráter globalizante em função da heterogeneidade ocasionada pelas formações profissionais distintas (AZEVEDO e DE VASCONCELLOS, 2013).

Após a construção da ementa, seus fundamentos foram abordados por meio do Núcleo Integrador em turmas do EMIEPT do IFNMG/Diamantina, onde se matricularam estudantes dos primeiros e segundos anos dos cursos técnicoprofissionalizantes em Informática, Meio Ambiente e Teatro, ao longo dos períodos letivos de 2018 e 2019.

Na sequência, o trabalho envolveu a elaboração e aplicação de formulários de aceitação em escala, em formato eletrônico, com o propósito de avaliar o nível de dificuldade demonstrado em um primeiro contato com uma LP, uma vez que os estudantes não apresentavam conhecimento prévio sobre a linguagem, Python. Esse estudo, envolveu a participação de 110 estudantes e ocorreu pela exploração dos seguintes aspectos da LP: variáveis, estruturas de repetição, estruturas de decisão, listas e ambiente de programação, onde os estudantes classificaram o nível de aprendizagem do curso em uma escala de ( 1 = muito fácil, $5=$ muito difícil $)$ para cada aspecto.

\section{Resultados e discussões}

A sistematização dos núcleos curriculares estruturantes do IFNMG/Diamantina exibe um cunho dinâmico, uma vez que permite a análise e o desenvolvimento constante de unidades formativas por meio do Núcleo Integrador. 
Programa de Pós-graduação em Educação, Universidade Federal do Ceará

Fortaleza-CE-Brasil

Disposição, que desencadeia reflexões contínuas e intrínsecas à elaboração de unidades com temáticas contemporâneas, desenvolvidas à luz dos anseios e aptidões projetados pela sociedade. Diante desse cenário formativo, nossa proposta de ementa (quadro 1), se integraliza como uma estratégia à introdução do pensamento computacional no EMIEPT.

Quadro 1. Proposta de ementa para abordagem de Linguagem de Programação

\begin{tabular}{|l|l|}
\hline UNIDADE CURRICULAR & Linguagem de Programação Python \\
\hline ANO & $1^{\circ}, 2^{\circ}, 3^{\circ}$ anos \\
\hline CARGA HORÁRIA & 20 horas \\
\hline
\end{tabular}

EMENTA: Resolução de problemas utilizando algoritmos e raciocínio lógico; conceitos básicos de ciência da computação através da linguagem de programação Python; conceitos e estruturas fundamentais de programação relacionados a tecnologia do dia a dia (expressões, operadores e variáveis, estrutura sequencial, estrutura de decisão, estruturas de repetição) e estruturas básicas de dados (Listas e Funções).

\section{BIBLIOGRAFIA}

$\mathrm{Na}$ construção do referencial teórico foram adotadas bibliografias acessíveis a iniciantes, e sugere-se três autores essenciais à boa condução da disciplina:

BORGES, L. E. (2014). Python para Desenvolvedores: Aborda Python 3.3. Novatec Editora.

MATHEUS E. (2017) Curso Intensivo de Python: Uma introdução prática e baseada em projetos à programação (Portuguese Edition) Novatec Editora.

MENEZES, N. N. C. (2016). Introdução à programação com Python-2 edição: Algoritmos e lógica de programação para iniciantes. Novatec Editora.

Fonte: Elaborado pelos autores

A ementa sugerida no Quadro 1 foi elaborada com vistas à construção do conhecimento no contexto da inserção tecnológica aplicada ao percurso formativo em um espectro profissional amplo (perfis profissionais distintos). Esta foi idealizada por meio dos aspectos relacionados no Plano de Ensino da Unidade (Quadro 2), os quais vislumbram o estímulo ao desenvolvimento de novas habilidades e visão múltipla das situações/realidade com consequente aplicação na dimensão profissional. 
Quadro 2. Itens do Plano de Ensino da Unidade Curricular Linguagem de Programação, Python

\begin{tabular}{|l|l|}
\hline ASPECTOS & \multicolumn{1}{|c|}{ ELUCIDAÇÃO } \\
\hline Competências & $\begin{array}{l}\text { Com este conhecimento os estudantes serão capazes de pensar } \\
\text { algoritmicamente, ou seja, no sentido de elaborar uma } \\
\text { sistematização em etapas bem definidas para a solução de } \\
\text { problemas do mundo real em linguagem computacional. }\end{array}$ \\
\hline Objetivos & $\begin{array}{l}\text { Relacionar os conceitos da LP com as tecnologias consumidas } \\
\text { no dia a dia, além de vislumbrar as potencialidades de uma LP } \\
\text { nas soluções envolvendo a ciência da computação nos desafios } \\
\text { profissionais. }\end{array}$ \\
\hline Metodologia & $\begin{array}{l}\text { Desenvolvimento do conteúdo programático por meio de aulas } \\
\text { práticas, expositivas e dialogadas. Com posterior aplicação do } \\
\text { conteúdo por meio da construção de projetos e abordagens } \\
\text { conjuntas entre os estudantes com diferentes perfis técnico- } \\
\text { profissionais. }\end{array}$ \\
\hline $\begin{array}{l}\text { Avaliação de } \\
\text { aprendizagem }\end{array}$ & $\begin{array}{l}\text { Como instrumentos de avaliação da aprendizagem foram } \\
\text { adotadas as dinâmicas em grupo }{ }^{5} \text {, as quais tiveram como } \\
\text { propósito aumentar a interação entre os estudantes e suscitar a } \\
\text { construção de projetos com abordagens amplas, pela integração } \\
\text { das áreas técnico-profissionais. }\end{array}$ \\
\hline
\end{tabular}

Fonte: Elaborado pelos autores

A análise dos requisitos de Farooq et al. (2012), a aspiração abrangente da proposta, tal como os estudos realizadas por Grandell et al. (2006) e Elker (2000), os quais sugerem a Python como linguagem ideal ao ensino de iniciantes na educação básica, foram determinantes para a escolha dessa linguagem (Python) em nossa proposta.

$\mathrm{Na}$ implementação da Python adotou-se o Trinket (https://trinket.io/python) como ambiente de programação (i.e. onde os estudantes efetivamente escrevem o código) visto sua facilidade de execução por meio do navegador da internet. Cabe salientar que o uso do navegador de internet torna a programação mais acessível,

Instrumento educacional no qual a aprendizagem acontece em um processo coletivo, onde o encontro de pessoas promove a construção conjunta do saber, estimula a capacidade criadora, aprimora a desenvoltura e a produtividade, revela a possibilidade de transformações, estimula o trabalho em equipe e pode melhorar as relações interpessoais e intrapessoais, possibilitando um caminho para se interferir na realidade, modificando-a (SBDG, 2006). 
uma vez que se excluem os desafios quanto à sua instalação/configuração.

No que se refere aos resultados da análise preliminar (tabela 1) de nossa proposta educacional, sugeridos por meio de formulários de aceitação em escala, depreende-se que houve uma boa aceitação dos estudantes quanto aos conceitos apontados pela ementa e LP (Python). Posto que, as médias dos aspectos avaliados estão situadas entre 1,8 e 3,44 na escala $1-5$, dados que indicam que nenhum aspecto foi considerado particularmente difícil pelos estudantes.

Tabela 1. Avaliação de vários aspectos na perspectiva do aluno $(1=$ muito fácil, $5=$ muito difícil)

\begin{tabular}{c|c}
\hline Aspecto avaliado & Média \\
\hline Variáveis & 2,13 \\
Estruturas de decisão & 2,58 \\
Estruturas de repetição & 3,44 \\
Listas & 2,88 \\
Ambiente de programação & 1,8 \\
\hline Média total & $\mathbf{2 , 5 7}$ \\
\hline
\end{tabular}

Fonte: Elaborado pelos autores

O ambiente de programação foi considerado um ponto positivo, segundo a avaliação dos estudantes (média de 1,8). Destaca-se que um ambiente de programação fácil e gratuito é fundamental no processo de democratização da aprendizagem.

Ainda sob a concepção de uma boa compreensão e assimilação pelos estudantes, salienta-se que neste caso o Trinket é livre, o que permite uma rápida demonstração de aspectos da programação propostos neste estudo (variáveis, estruturas de repetição, estruturas de decisão e listas) tornando a execução do código dinâmica e compreensível. Além disto, sob o aspecto do ensino de Listas, a Python oferece uma boa expressividade ao introduzir tipos de dados compostos de forma simplificada (quadro 3). 
Quadro 3. Comparação de programas escritos em Java e Python

\begin{tabular}{|c|c|c|}
\hline \multirow{2}{*}{$\begin{array}{l}\text { Conceito } \\
\text { ensinado }\end{array}$} & \multicolumn{2}{|c|}{ Exemplos de implementação dos conceitos } \\
\hline & Java & Python \\
\hline Variáveis & $\begin{array}{c}\text { public class ExampleClass } \\
\{ \\
\text { public static void } \\
\text { main(String[]args) } \\
\text { int } x=42 \\
\text { System.out.println }(\mathrm{x}) \\
\}\end{array}$ & $\begin{array}{l}x=42 \\
\text { print } x\end{array}$ \\
\hline $\begin{array}{c}\text { Estruturas } \\
\text { de controle/ } \\
\text { decisão }\end{array}$ & 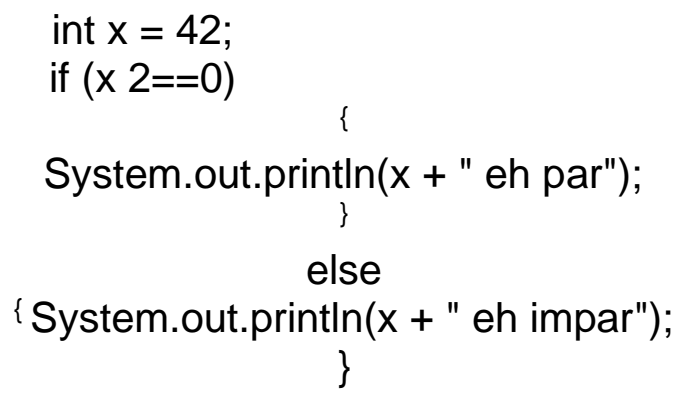 & $\begin{array}{l}x=42 \\
\text { if } x 2==0 \text { : } \\
\text { print } x, \text { "eh par" } \\
\text { else: } \\
\text { print } x \text {, "eh impar" }\end{array}$ \\
\hline $\begin{array}{l}\text { Estruturas } \\
\text { de } \\
\text { Repetição }\end{array}$ & $\begin{array}{c}\text { for (int } \mathrm{i}=\underset{\{}{0} ; \mathrm{i}<42 ; \mathrm{i}++) \\
\text { System.out.print } \ln (\mathrm{i}) \\
\}\end{array}$ & $\begin{array}{c}\text { for } i \text { in range } \\
\text { print } i\end{array}$ \\
\hline
\end{tabular}

Fonte: Adaptado de Koulouri, Lauria \& Macredie (2015)

Em referência ao cotidiano da sala de aula, observou-se que a Python apresenta características ideais ao ambiente diversificado e introdutório a que se propõe: alto nível (i.e os comandos são parecidos com a linguagem humana), interpretada (pode executar um comando de cada vez), com tipagem dinâmica (que não exigem declarações do tipo de dado da variável, sendo exemplo de tipos de dados: valores inteiros, reais, lógicos) e legibilidade do código. Sua sintaxe intuitiva e limpa se comparada a outras linguagens de programação (quadro 3), pode ser interpretativa proporcionando um feedback imediato, suscitando rapidez na demonstração dos conceitos de programação e detecção de erros na codificação, comuns em programadores iniciantes. Além de impor uma forma recortada 

é aberto e amplamente utilizado com documentação detalhada e disponível na web, com uma comunidade engajada de usuários (MILLMAN \& AIVAZIS, 2011; PERKEL, 2015).

Considerando o contexto da sala de aula sobre o ponto de vista da verificação da aprendizagem, averiguou-se que as dinâmicas em grupo (formadas por estudantes pertencentes a cursos e/ou eixos distintos) proporcionaram uma interação singular entre os estudantes, o que alavancou a construção de projetos multidisciplinares e direcionou os distintos perfis profissionais a apresentarem propostas para uso da Python que abordaram desde automatização de tarefas de cálculos até estrutura de dados mais sofisticadas como Listas (i.e conjuntos de números, variáveis, tarefas, ou qualquer elemento que se possa enumerar e formar um conjunto, estrutura importante para a construção de algoritmos mais bem elaborados).

Face à essa boa interação dos estudantes com a LP, reiteramos a importância da compreensão dos recursos aportados pela tecnologia pelos mais diversos perfis profissionais, visto a aplicabilidade da programação em diversas áreas de conhecimento. A exemplo, citamos o crescimento exponencial do volume de dados ambientais disponíveis (como dados disponibilizados por novos sensores e satélites que monitoram o ecossistema), os quais precisam ser manipulados por meio de uma LP, circunstância que torna esse conhecimento fundamental aos estudantes dos cursos técnicos em meio ambiente.

Finalizamos este tópico sob o entendimento de que a programação se tornou uma competência central dirigida à múltiplas áreas, um tipo de alfabetização básica da era digital para que os sujeitos sejam capazes de compreender toda tecnologia ao seu redor. E nesse sentido, consideramos que a inclusão de uma LP no nível médio, trata-se de um importante instrumento nos processos de formação profissional, além de introduzir o dinamismo do mundo e mercados atuais no ambiente escolar.

\section{Considerações finais}

A inserção de uma parte diversificada, prevista nos currículos dos cursos do EMIEPT do IFNMG/Diamantina cria, por meio da oferta de unidades curriculares 
no Núcleo Integrador, um espaço para o desenvolvimento de novos estímulos cognitivos e reflexões. Uma abordagem que fomenta o diálogo entre o currículo e as demandas emergentes, ao passo em que nos remete a um universo onde as práticas pedagógicas se entrelaçam aos artifícios sociais e suas modificações constantes oriundas de diferentes tempos, histórias e espaços (SILVA \& DE OLIVEIRA PAVÃO, 2018).

Segundo essa compreensão acerca da importância do currículo no processo formativo, torna-se imprescindível considerar o pensamento computacional na formação estudantil, visto o impacto e a presença das tecnologias digitais em todos os aspectos da sociedade. Desse modo, a criação de uma ementa para o ensino de LP no nível médio, direcionada a cursos que integram diferentes eixos tecnológicos, representa uma estratégia somatória ao processo de ensino e aprendizagem, no sentido de criar condições para que os estudantes sejam capazes de elucidar como as tecnologias digitais funcionam, e pela apropriação desses conceitos tornarem-se aptos a aplicá-las em diferentes contextos e situações.

Por fim, no sentido de fundamentar a viabilidade da presente proposta de ensino de LP no nível médio, ressalta-se que o resultado dos formulários de aceitação indicou boa interação e assimilação do conteúdo entre os estudantes, que se mostraram capazes de ressignificar o conteúdo adquirido em sala de aula por meio da construção de propostas de trabalho destinadas a transformação do cotidiano pela aplicação no mundo do trabalho. Adicionalmente, a aceitação satisfatória pelos estudantes se mostrou indiferente ao perfil profissional, o que sugere como adequados a escolha da Python, enquanto LP, bem como a construção dos processos contemplados em nossa ementa curricular.

\section{Referências Bibliográficas}

ANTUNES, Ângela; PADILHA, Paulo Roberto. Educação cidadã, educação integral: fundamentos e práticas. São Paulo: Instituto Paulo Freire, 2000, p.23-102.

AZEVEDO, Diego Napoleão Viana; DE VASCONCELLOS, Maria Lúcia Barbosa. Formação de tradutores: a Contribuição da terminologia para uma proposta de desenho de ementa de disciplina a partir de abordagem por tarefa de tradução. Traduzires, v. 2, n. 2, p. 41-52, 2013.

BEHRENS, Marilda Aparecida; JOSÉ, Eliane Mara Age. Aprendizagem por projetos e os contratos didáticos. Revista Diálogo Educacional, v. 2, n. 3, p. 1-19, 2001. 
BRASIL.Lei no 9.394, de 20 de dezembro de 1996. Estabelece as diretrizes e bases da educação nacional. Brasília, DF: Presidência da República, 1996. Disponível em:<http://www.planalto.gov.br/ccivil 03/leis//9394.htm>. Acesso em: 11 de novembro de 2019.

BRASIL. Resolução № 6, de 20 de setembro de 2012. Define Diretrizes Curriculares Nacionais para a Educação Profissional Técnica de Nível Médio. Diário Oficial da União. Brasília: DF. Disponível em:<http://portal.mec.gov.br/index.php?option=com docman\&vie $\mathrm{W}=$ download\&alias=11663-rceb006-12-pdf\&category slug=setembro-2012pdf\&ltemid=30192>Acesso em: 20 de outubro de 2019.

BRASIL. Lei no 13.415, de 16 de fevereiro de 2017. Altera as Leis $\mathrm{n} \cong 9.394$, de 20 de dezembro de 1996. Diário Oficial da União. Brasília: DF. Disponível em: $<$ http://www.planalto.gov.br/ccivil 03/ ato2015-2018/2017/lei//13415.htm>. Acesso em: 11 de novembro de 2019.

BORGES, L. E. Python para Desenvolvedores: Aborda Python 3.3. Novatec Editora,, 2004, p. 20-289.

CIAVATTA, Maria; RAMOS, Marise. Ensino Médio e Educação Profissional no Brasil: dualidade e fragmentação. Retratos da Escola, v. 5, n. 8, p. 27-41, 2012.

CONIF. FDE. Diretrizes indutoras para a oferta de cursos técnicos integrados ao Ensino Médio na Rede Federal de Educação Profissional, Científica e Tecnológica (2018). Disponível em: $<$ https://www.ifsc.edu.br/documents/38101/1079513/Diretrizes +EMl++ Reditec2018.pdf/0cd97af4-bad5-b347-4869-7293ac87eb69>. Acesso em 04 de novembro de 2019.

COSTA JÚNIOR, José Geraldo Bastos da, SOUZA, Francisca Leidiana de, SANTOS, Luiz Antônio da Silva \& SOUZA, Francisca das Chagas Silva. O currículo integrado nos documentos do IFRN: perspectivas e desafios. Revista Labor, v.2, n.18, p.180192, 2017.

DA SILVA, Claudia Sebastiana Rosa \& PORTILHO, Evelise Maria Labatut. Cultura Escolar, Formação Continuada E Estratégias De Ensino. Revista e-Curriculum, v.16, n.3,p. 911-933, 2018.

DA SILVA, Karen Cristina Jensen Ruppel; DE FÁTIMA FLACH, Simone. Educação Integral: Em Defesa De Uma Concepção Emancipatória. Revista Educativa-Revista de Educação, v. 20, n. 3, p. 717-737, 2017.

DA SILVA, Thiago Reis; LOPES, Ranyer; MEDEIROS, Taina Jesus; ARANHA, Eduardo; MEDEIROS, Handerson. Ensino-aprendizagem de programação: uma revisão sistemática da literatura. Revista Brasileira de Informática na Educação, v. 23, n. 1, p.182-196, 2015. 
ELKNER, Jeffrey. Using Python in a high school computer science program. In: Proceedings of the 8th International Python Conference, p. 2000-2001, 2000.

FAROOQ, Muhammad Shoaib et al. A Qualitative Framework for Introducing Programming Language at High School. Journal of Quality and Technology Management, v. 8, n. 2,p. 135-151, 2012.

FEIJÓ, Jerciano Pinheiro; XAVIER, Antônio Eudes; ARRAIS NETO, Enéas De Araújo. A educação como instrumento de transformação da realidade. Revista Labor, v. 1, n. 22, p. 103-113, 2019.

FLACH, Simone Fátima; SCHLESENER, Anita Helena. Análise de conjuntura sobre a ocupação de escolas no Paraná a partir do pensamento de Antonio Gramsci. ETDEducação Temática Digital, v. 19, n. 1, p. 165-186, 2017.

FREIRE, Paulo. Extensão ou Comunicação. $7^{a}$ ed. Rio de Janeiro, Paz e Terra,1969, p.25-117.

FERRETI, Celso João; SILVA, Monica Ribeiro da. Reforma do ensino médio no contexto da medida provisória n. 746/2016: estado, currículo e disputas por hegemonia. Educação \& Sociedade, v. 38, n. 139, p. 385-404, 2017.

FRIGOTTO, Gaudêncio; CIAVATTA, Maria; RAMOS, Marise. O trabalho como princípio educativo no projeto de educação integral de trabalhadores. Excertos, v. 9, p. 1-14, 2005. Disponível em:<http://redeescoladegoverno. fdrh. rs. gov. br/upload/1392215839> Acesso em: 14 de novembro de 2019.

GARDNER, Howard, CHEN, Jie-qi \& MORAN, Seana. Inteligências múltiplas. Penso Editora, 2009, p.109-425.

GRANDELL, Linda et al. Why complicate things?: introducing programming in high school using Python. In: Proceedings of the 8th Australasian Conference on Computing Education-Volume 52. Australian Computer Society, Inc., p. 71-80,2006.

IFNMG, Instituto Federal de Educação, Ciência e Tecnologia do Norte de Minas Gerais (2017). Projeto pedagógico de curso. Disponível em: $<$ http://documento.ifnmg.edu.br

/action.php?kt path info=ktcore. actions. document.view\&fDocumentld=22345>.

Acesso: 10/novembro/2018.

KOULOURI, Theodora; LAURIA, Stanislao; MACREDIE, Robert D. Teaching introductory programming: A quantitative evaluation of different approaches. ACM Transactions on Computing Education (TOCE), v. 14, n. 4, p. 1-28, 2014.

MATHEUS E. Curso Intensivo de Python: Uma introdução prática e baseada em projetos à programação (Portuguese Edition) Novatec Editora, 2017, p. 99-685.

MENEZES, N. N. C. Introdução à programação com Python-2 $2^{\underline{a}}$ edição: Algoritmos e lógica de programação para iniciantes. Novatec Editora, 2016, p.27-324. 
MILLMAN, K. Jarrod; AIVAZIS, Michael. Python for scientists and engineers. Computing in Science \& Engineering, v. 13, n. 2, p. 9-12, 2011.

MITCHEL RESNICK, J. M. Scratch: Programming for Everyone, Communications of the acm, v. 52, n. 11, p. 52-67, 2009.

PALFREY, John Gorham; GASSER, Urs. Born digital: Understanding the first generation of digital natives. Read How You Want. com, 2011.

PERKEL, Jeffrey M. Programming: pick up Python. Nature News, v. 518, n. 7537, p. 125, 2015.

PINTO, A. C., et al. Conceito de ser humano de professores do curso de enfermagem à luz do homo complexus de Edgar Morin/Human concept of teachers of nursing course in the light of edgar morin homo complexus. Brazilian Journal of Development, v.5, n.9, p.14543-14555, 2019.

RAMOS, Marise. Concepção do ensino médio integrado. Texto apresentado em seminário promovido pela Secretaria de Educação do Estado do Pará nos dias, v.8,p. 1-26, 2008. Disponível em: <http://forumeja.org.br/go/sites/forumeja.org.br. go/files/concepcaodoe $\mathrm{n}$ sino medio integrado5.pdf >. Acesso em: 16 out. 2019.

SOCIEDADE BRASILEIRA DE DINÂMICAS DE GRUPO (SBDG). Dinâmica de grupo: conhecendo a história da dinâmica dos grupos no Brasil. Blumenau: SBDG, 2006.

SILVA, Mariane Carloto; DE OLIVEIRA PAVÃO, Silvia Maria. Possibilidades das adaptações curriculares na educação superior. Revista e-Curriculum, v. 16, n. 3, p. 621-649, 2018.

SCAICO, Pasqueline Dantas et al. Teaching Programming in High School: an approach guided by design with Scratch language. Brazilian Journal of Computers in Education, v. 21, n. 02, p. 92, 2013.

SCHOOLNET, Europian. Computing our future. computer programming and coding priorities, school curricula and initiatives across europe. Technical report European Schoolnet, 2014.

TABILE, Ariete Fröhlich; JACOMETO, Marisa Claudia Durante. Fatores influenciadores no processo de aprendizagem: um estudo de caso. Revista Psicopedagogia, v. 34, n. 103, p. 75-86, 2017.

VALENTE, José Armando. Integração do pensamento computacional no currículo da educação básica: diferentes estratégias usadas e questões de formação de professores e avaliação do aluno. Revista e-Curriculum, v. 14, n. 3, p. 864-897, 2016. 


\section{Bruno Lopes de Faria}

Diamantina, Minas Gerais, Brasil.

Professor de Informática no Instituto Federal do Norte de Minas Gerais (IFNMG) campus Diamantina. Possui graduação em Ciência da Computação pela Universidade Federal de Viçosa (UFV) e Mestrado em Meteorologia pela UFV, e é doutorando em Ciências Florestais pela UFVJM. Atualmente coordenador dos cursos técnicos em informática do IFNMG campus Diamantina.

E-mail: bruno.lopes@ifnmg.edu.br

Link do Lattes: http://lattes.cnpq.br/9314295919473349

\section{Janainne Nunes Alves}

Diamantina, Minas Gerais, Brasil.

Doutora em Físico-química pela Universidade Federal de Uberlândia (UFU). Atualmente professora no Instituto Federal de Educação, Ciência e Tecnologia do Norte de Minas Gerais, onde exerce a função de Coordenadora de Pesquisa, Pós-Graduação e Inovação do Campus Diamantina.

E-mail: janainne.alves@ifnmg.edu.br

Link do Lattes: http://lattes.cnpq.br/7116639699446494

\section{Claudiane Moreira Costa}

Diamantina, Minas Gerais, Brasil.

Possuo graduação em Licenciatura em Matemática pelo Instituto Federal de Educação Ciência e Tecnologia do Norte de Minas Gerais (2011). Pós -Graduação em Gestão em Estatística e Matemática Aplicada pela FAVAG(2012). Mestranda no Programa de Pós-Graduação em Educação Profissional e Tecnológica - ProfEPT . Atualmente ocupo o cargo de Técnica em Assuntos Educacionais no IFNMG Campus Diamantina.

E-mail: claudiane.costa@ifnmg.edu.br

Link do Lattes: http://lattes.cnpq.br/1732506655828239

\section{Recebimento: 17/05/2020 \\ Aprovação: 04/03/2021}

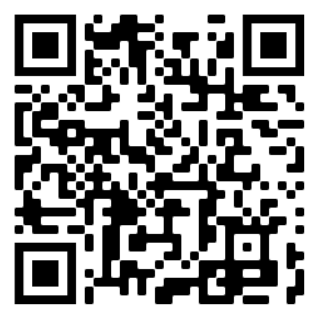

\section{Q. Code}

\section{Editores-Responsáveis}

Dr. Enéas de Araújo Arrais Neto, Universidade Federal do Ceará, UFC, Ceará, Brasil

Dr. Sebastien Pesce, Universidade de Orléans, França 